\title{
Sociologie et histoire : la contribution de Durkheim
}

Sociology and history: Durkheim's contribution

\section{Massimo Borlandi}

\section{(2) OpenEdition}

Journals

Édition électronique

URL : http://journals.openedition.org/ress/3920

DOI : $10.4000 /$ ress.3920

ISBN : 1663-4446

ISSN : $1663-4446$

Éditeur

Librairie Droz

Édition imprimée

Date de publication : 15 décembre 2017

Pagination : 83-104

ISSN : 0048-8046

Référence électronique

Massimo Borlandi, «Sociologie et histoire : la contribution de Durkheim », Revue européenne des sciences sociales [En ligne], 55-2 | 2017, mis en ligne le 15 décembre 2020, consulté le 04 janvier 2021. URL : http://journals.openedition.org/ress/3920 ; DOI : https://doi.org/10.4000/ress.3920 


\title{
SOCIOLOGIE ET HISTOIRE: LA CONTRIBUTION DE DURKHEIM
}

MASSIMO BORLANDI

Université de Turin - DCPS

mgb.borlandi@gmail.com

Résumé. Selon Durkheim, un phénomène social n'est expliqué que si l'on remonte à ses origines. Cela met en lumière la façon dont il conçoit les relations entre la sociologie et l'histoire. Une attention particulière vise à cerner ce que Durkheim comprend par «méthode comparative».

Mots-clés: Émile Durkheim, histoire, méthode comparative, sociologie.

\begin{abstract}
According to Durkheim, a social phenomenon cannot be explained without going back to its origins. This sheds light on the way he perceives the relationships between sociology and history. This article aims in particular at clarifying what Durkheim means by "comparative method".
\end{abstract}

Keywords: comparative method, Émile Durkheim, history, sociology. 
Les relations entre la sociologie et l'histoire, si controversées depuis qu'on en parle, sont réglées par les deux sens que le mot «histoire » prend dans les principales langues. Ce terme désigne tant le passé, c'est-à-dire l'accumulation des faits qui nous précèdent (les res gestae des Latins), que le récit de ces mêmes faits, c'est-à-dire la discipline qui les étudie (l'historia rerum gestarum, l'histoire profession des historiens). Si on met en rapport la sociologie avec l'histoire au sens du passé, on est amené à se demander quelle est la part qui revient au passé dans l'explication des phénomènes sociaux. Un phénomène social, tel qu’on le connaît aujourd'hui, est-il l'aboutissement de ce qu'il était hier? Faut-il, pour en rendre compte, se renseigner sur ses états antérieurs? Par contre, si on met en rapport la sociologie avec l'histoire au sens de la discipline qui étudie le passé, ce rapprochement consiste à montrer sous quels aspects les deux savoirs diffèrent. Qu'est-ce qui sépare les métiers de sociologue et d’historien?

Émile Durkheim discute ces deux manières de relier la sociologie à l'histoire, qu'il mélange somme toute moins que d'autres savants, dans une trentaine de textes et à différentes occasions. Ses certitudes sont les suivantes. La part qu'il faut faire au passé dans l'explication sociologique est grande, d'autant plus grande que les phénomènes à expliquer sont des institutions, i.e. des normes de comportement et des croyances constituées par les collectivités. «Science des institutions» (Durkheim, 1947 [1895], p.XXII), la sociologie ne les aura pas vraiment comprises tant qu'elle n'aura pas établi d'où elles viennent, comment elles sont nées. Une «méthode comparative» (sans conteste le chapitre le plus complexe de l'œuvre de Durkheim) permettra au sociologue de traduire en procédés vérifiables l'étude de la genèse des institutions ainsi envisagée. Quant aux différences, ou aux ressemblances, entre la sociologie et l'histoire récit, Durkheim distingue l'objet et la méthode de cette dernière. L’objet, ce sont des événements particuliers, propres à un endroit ou à une époque donnés, ou bien des faits généraux, i.e. répandus. La méthode est l'érudition. Un fait général étant une institution, l'originalité de la sociologie par rapport à l'histoire est destinée à se réduire à mesure que l'histoire s'intéresse aux faits généraux et délaisse les événements particuliers. De plus, c'est seulement grâce à l'érudition des historiens que les 
sociologues comparatistes peuvent espérer atteindre des résultats - cas non rare d'une méthode qui en implique une autre.

Je présenterai les thèses de Durkheim sur l'explication sociologique conçue comme recours au passé dans les sections I-3 et celles sur les convergences de la sociologie et de l'histoire dans la section 4 .

\section{I.EXPLIQUER, EN SOCIOLOGIE, C'EST REMONTER AU PASSÉ}

C'est dans la leçon d'ouverture du cours de i888-1889 sur la famille, deuxième cours public de Durkheim à Bordeaux, que l'idée que le passé éclaire le présent (aussi ancienne que l'idée contraire, selon laquelle le présent est la clé du passé) prend la valeur d'un axiome fondateur. Durkheim informe que le recensement des types de famille auquel il va se consacrer, pour la plupart disparus, n'est pas une fin en soi. Bien au contraire, «de cette étude du passé se dégagera une explication du présent qui deviendra de plus en plus complète, à mesure que nous avancerons dans nos recherches.» (1888b, p. I5). En effet, «la famille moderne contient en elle-même, comme en raccourci, tout le développement historique de la famille», de sorte que, pour chaque espèce familiale identifiée, on essaiera d'établir «ce qu'elle peut avoir de commun avec la famille d’aujourd'hui et ce qu'elle en explique.» (ibid.). «Si loin que nous remontions dans le passé nous ne perdons jamais le présent de vue. » (ibid., p. 16).

De même, la préface du volume 2 de L’Année sociologique énonce que «Pour comprendre le présent, il faut en sortir. » (I899, p. I39) et le cours de 1904-1905 sur l'Histoire de l'enseignement secondaire en France maintient que «c'est dans le passé que l’on trouve les éléments dont est formé le présent» (I969b [1938], p. 2I). Séparés d’une dizaine d’années, le mémoire sur «La prohibition de l’inceste» et l'article «Sociologie religieuse et théorie de la connaissance» (qui finira en partie dans l'introduction des Formes élémentaires de la vie religieuse) assurent que la détermination du «point de départ» est la condition de toute explication sociologique réussie, sachant qu'à défaut de saisir ce point, il va de soi qu’il s'agira au moins de tâcher de s'en approcher. 
[P] our bien comprendre une pratique ou une institution, une règle juridique ou morale, il est nécessaire de remonter aussi près que possible de ses origines premières; car il y a, entre ce qu'elle est actuellement et ce qu'elle a été, une étroite solidarité. Sans doute comme elle s'est transformée chemin faisant, les causes dont elle dépendait dans le principe ont elles-mêmes varié; mais ces transformations, à leur tour, dépendent de ce qu'était le point de départ. Il en est des phénomènes sociaux comme des phénomènes organiques; si le sens dans lequel ils doivent se développer n’est pas fatalement prédéterminé par les propriétés qui les caractérisent à leur naissance, celles-ci ne laissent pas d’avoir une influence profonde sur toute la suite de leur développement. (I898b, p. 37).

Toutes les fois [...] qu'on entreprend d'expliquer une chose humaine, prise à un moment déterminé du temps - qu'il s'agisse d'une croyance religieuse, d'une règle morale, d'un précepte juridique, d'une technique esthétique, d'un régime économique - il faut commencer par remonter jusqu'à sa forme la plus primitive et la plus simple, chercher à rendre compte des caractères par lesquels elle se définit à cette période de son existence, puis faire voir comment elle s'est peu à peu développée et compliquée, comment elle est devenue ce qu'elle est au moment considéré. Or, on conçoit sans peine de quelle importance est, pour cette série d'explications progressives, la détermination du point de départ auquel elles sont suspendues. C'était un principe cartésien que, dans la chaîne des vérités scientifiques, le premier anneau joue un rôle prépondérant. (1909b, p. 735-736; 1912, p. 4-5).

\section{Comment se conformer à cette recommandation? Comme suit.}

Tout phénomène social d'une certaine étendue et durée découle d'un processus de composition qui consiste dans le fait que des éléments nouveaux se sont progressivement ajoutés à des éléments préexistants. La première chose à faire est de décomposer le tout ainsi formé pour qu’il soit possible d'en considérer les éléments séparément l'un de l'autre, c'est-à-dire les dater et évaluer l’apport de chacun d'eux à la modification de l'ensemble, leur importance, et avant tout leur taille, pouvant avoir augmenté ou diminué au fil du temps. Cela signifie que, pour chaque composante du phénomène à expliquer, il faut identifier la cause qui en a provoqué l'apparition et voir si cette cause a continué d'agir depuis, ou si elle perdu de sa vigueur. Au début, l'identification d'une cause prend la forme d'une concurrence entre des conjectures jusqu'à ce que l'on voie que la mise en rapport de l'élément concerné avec une cause présumée précise (cet antécé- 
dent-là et aucun autre) donne des résultats : le premier change comme la seconde et au même moment qu'elle, ce qui prouve qu'il en est une conséquence. La cause recherchée est alors identifiée. On répète l'opération pour chaque élément du phénomène social en question. L'élément originaire sera évidemment le plus ancien rencontré et on désignera comme cause du phénomène la résultante de la façon dont se combinent les causes de ses composantes.

Dira-t-on, à ce point, que le phénomène est expliqué ? Absolument pas, car les liaisons causales établies pourraient être fortuites et doivent être vérifiées en élargissant l'observation à la marche du même phénomène dans d'autres sociétés. Plus ce phénomène persiste depuis longtemps - et c'est bien le cas des normes juridiques, des mœurs et des croyances majeures qui disciplinent notre vie et qui sont toutes des legs de civilisations antérieures révolues -, plus il est opportun que l'observation soit menée sur des sociétés à la fois nombreuses et hétérogènes, c'est-à-dire appartenant à différents types (ou «espèces »), et qui, de préférence, se soient succédé. C'est seulement après qu’elle aura été corroborée par de tels rapprochements qu'une explication pourra être considérée comme valide. Des raccourcissements de ce procédé sont admis. On peut se borner à analyser des sociétés homogènes (du même type) si on est sûr que le phénomène à expliquer leur est contemporain (i.e., est né avec elles ou aussitôt après leur formation), alors que toute conclusion tirée de l'observation d'une seule société n'est acceptable que lorsque le phénomène visé est relativement simple et irrégulièrement distribué sur la surface d'un pays, de sorte que sa mise en rapport avec des données agrégées locales (des variables «écologiques» dans le vocabulaire de la sociologie post-durkheimienne) serait tout de même explicative².

Rangé sous le nom de «méthode comparative», exposé dans le chapitre VI des Règles touchant à l'administration de la preuve (1947 [1895], p. I34-I38), repris dans l'article «Sociologie et sciences sociales» (1909a, p. 156) et rappelé ici ou là (I898a, p. 32 et 35 ; 190I, p. 294-295; 1903, p. 196), ce précis des opérations à accomplir pour parvenir à la meilleure des explications sociologiques

2 C'est le cas de phénomènes moraux tels la natalité, la nuptialité et le crime, qui relèvent de l'intensité de certains «courants d'opinion» et varient selon les villes ou les campagnes, les professions, l'âge et l'état civil. 
possibles - explication axée sur la détermination du premier anneau de la chaîne - est esquissé encore dans la première leçon du cours sur la famille de I888-1889. La famille, décomposée dans ses éléments (consanguinité, mariage, descendance, etc.), est, en effet, l'institution à l'étude de laquelle Durkheim s'applique le plus (Davy, 1925; Lamanna, 2002), avec les «règles de conduite sanctionnées», sujet du cours maintes fois réitéré sur la «physique» ou la «physiologie» des mœurs et du droit (1969c [1950]) 3. Ce n'est cependant pas à l'occasion de la leçon d'ouverture de décembre i 888 que les mots «méthode comparative» font leur apparition dans l'œuvre de Durkheim (I888b, p. 3 I). Il les a déjà employés au début de l’année scolaire précédente: décembre I887. «Chargé d'enseigner une science née d'hier et qui ne compte encore qu'un petit nombre de principes définitivement établis » (1888a, p. 77), Durkheim n’hésite pas à inclure parmi ces principes «l'expérimentation indirecte, en d'autres termes la méthode comparative» (ibid., p. ıoo - c'est moi qui souligne), montrant qu’il a dès le début les idées claires sur ce point. Même au sujet des idées de Durkheim, l'on peut dire qu'on les comprend d'autant mieux qu'on en connaît les sources.

\section{LA MÉTHODE COMPARATIVE}

«Expérimentation indirecte» figure dans le Cours de philosophie positive, leçons 40 et 48. Auguste Comte caractérise ainsi l'«analyse pathologique» ou étude des maladies, aussi bien des organismes vivants que des sociétés (les crises politiques), qu'il conçoit comme une façon de remédier aux difficultés de l'expérimentation respectivement en biologie et en sociologie (Comte, I975 [I830-I842], vol. I, p. 696-698, 74I ; vol. 2, p. I42-I44). Dans les sciences, l'expérimentation proprement dite n'est que directe et signifie que les faits dont la répétition sert à tester une hypothèse sont provoqués. Mais comment les provoquer dès lors qu'il s'agit d'intervenir sur l'organisme individuel et l'organisme social? Durkheim, qui en décembre I887 semble suivre Comte - lequel est convoqué i 8 fois avant le passage que je viens de citer -, donne l'air d'inventer, lui, l'expression «expérimentation indirecte» dans sa leçon d'ouverture de décembre I888 (I888b, p. I4) 
où il s'appuie sur les vues de Claude Bernard en matière d'expérience (ibid., p. I3), alors que, huit ans après, dans Les Règles, c'est le John Stuart Mill du System of Logic qu'il sollicite sur le même sujet. Mill, en assurant à son tour que l'expérimentation est impossible dans la science sociale, exclut que ses quatre méthodes d'investigation expérimentale, dont celle des variations concomitantes, y soient applicables. La limite à la transposabilité de cette dernière méthode résiderait notamment dans le fait qu'un phénomène social est toujours l'effet d'une pluralité de causes (Mill, 1974 [I843], p. 88I-8844).

Durkheim objecte qu'en sociologie les faits permettant de tester des hypothèses s'offrent à l'observation - il suffit de savoir les repérer et les grouper (c'est bien en ce sens que les expériences du sociologue peuvent être qualifiées d'indirectes) - et que, soit le principe de causalité n'est pas univoque, soit la règle qui pose qu'à un même effet correspond toujours une même cause doit valoir également en sociologie. Après quoi, plus rien ne l'empêche de présenter sa méthode comparative comme étant le procédé qui consiste à établir que deux phénomènes évoluent parallèlement (parallélisme vérifié par l'étude de leurs variations concomitantes mise ainsi à l'abri de la remarque de manquer de caractère expérimental) et que leur relation (leur liaison causale) revient dans de nombreux contextes la plupart du temps (I947 [I895], p. I24-I32). «On cherchera d'abord, à l'aide de la déduction, comment l'un des deux termes a pu produire l'autre; puis on s'efforcera de vérifier le résultat de cette déduction à l'aide d'expériences, c'est-à-dire de comparaisons nouvelles. » (ibid., p. Izo-Iz I).

Finalement, chez Durkheim, comparer veut dire deux choses : rapprocher un effet de sa cause conjecturée (un sens des mots «comparer» et «comparaison » somme toute inusité et qui est on ne saurait plus périmé) ; rapprocher la relation (Durkheim écrit plutôt, avec Mill, la «variation») ainsi obtenue à d'autres relations (variations). 
Même l'expression «méthode comparative »- donc l'appellation de ce par quoi se traduit l'expérimentation indirecte - est d'origine comtienne et figure dans les leçons 40 et 48 du Cours d’ailleurs. Comte fait de la comparaison un troisième mode de l'exploration scientifique, après l'observation et l'expérimentation. La comparaison convient d'abord à la biologie. Retenu par la sociologie, ce procédé change de peau et devient la «méthode historique» par laquelle le sociologue rapproche, marginalement, les «divers états co-existants de la société humaine sur [...] la surface terrestre » (Comte, I975 [1830-1842], vol. 2, p. I46), et, principalement, «les divers états consécutifs de l'humanité » reliés entre eux par des rapports de «filiation» (ibid., p. I48-154, I72). Cette fois, dans les Règles, Durkheim cite Comte. Il lui est impératif, comme je vais le montrer, de prendre ses distances avec un tel procédé, en sorte que, s'il reconnaît avoir emprunté au fondateur du positivisme l'expression «méthode comparative » , c'est pour mieux se défendre d'en avoir adopté la méthode ${ }^{6}$. On est cependant fondé à suggérer qu’en I887-1888 l'«expérience comparative» et l'«expérimentation comparative» de Claude Bernard (I984 [I865], p. I8I-I85, 253-255) ont pesé dans la décision de Durkheim de se doter d'une méthode comparative?

Pour résumer, remonter au passé d’un phénomène social afin d'en éclairer le présent, c'est reconstruire l'enchaînement des causes (i.e. des antécédents concomitants) dont les changements résultent à chaque fois d'effets non accidentels. La multiplication, théoriquement illimitée, des comparaisons que le procédé de Durkheim requiert vise à démontrer le caractère régulier, et en ce sens nécessaire, des liaisons causales que la sociologie découvre.

5 «Puisque, d'autre part, les phénomènes sociaux échappent évidemment à l'action de l'opérateur [allusion à l'impossibilité de l'expérimentation directe], la méthode comparative est la seule qui convienne à la sociologie. Comte, il est vrai, ne l'a pas jugée suffisante; il a trouvé nécessaire de la compléter par ce qu'il nomme la méthode historique.» (1947 [1895], p. 124).

6 À remarquer que le Lalande ne retient que la méthode comparative de Durkheim.

7 II est question de la comparaison comme biais permettant de surmonter l'impossibilité de la méthode expérimentale (experimentalis methodus) en sociologie dans la thèse latine de Durkheim également (1892, p. 96). 


\section{DEUX ARGUMENTS DES RÈGLES}

Durkheim qualifie aussi sa méthode de «génétique (I947 [1895], p. I37 ; I90I, p. 295), et, plus tard, d'«historique et comparative» (I906b, p. I26) et d'«historique et objective» (1908b, p. 59 $)$. À trois reprises, «histoire comparée» relaie «méthode comparative» (1900a, p. I26; I90I, p. 295; I909a, p. I53). L'incitation de Durkheim à comparer n'en demeure pas moins parfois assez vague dans la mesure où il ne détaille pas toujours l'ensemble des opérations, laborieuses, qu'il souhaite voir accomplies; sans compter que, à la fin, il est le premier à transgresser ses propres prescriptions, comme dans les Formes élémentaires où il compare en profondeur mais non pas en extension, puisque son analyse se borne à l'étude de quelques sociétés australiennes. Ces hésitations ne portent pas préjudice à la place que la méthode comparative occupe dans la hiérarchie des règles durkheimiennes de la méthode. Elle en est fermement au sommet: «La sociologie comparée n’est pas une branche particulière de la sociologie; c'est la sociologie même, en tant qu'elle cesse d'être purement descriptive et aspire à rendre compte des faits.» (1947 [1895], p. I37); «On n'explique qu'en comparant. » (I897a, p. I) ; «La méthode comparative est [...] l'instrument, par excellence, de la méthode sociologique.» (I909a, p. 156).

La prééminence que Durkheim assigne à la méthode comparative soulève le problème de la compatibilité des énoncés par lesquels il la défend avec deux arguments dont il fait état, notamment dans les Règles. Le premier est que le passé est pour peu de choses dans le cours de l'histoire. Durkheim est-il donc en contradiction avec ses propres propos? Le second argument est que les phénomènes sociaux remplissent le plus souvent des fonctions, i.e. des fins utiles, qu'on ne saurait négliger dans l’analyse. La fonction concurrence-t-elle donc la cause dans l'explication sociologique? Ces deux arguments sont de différente valeur. Le premier fait difficulté depuis toujours (voir Davy, 1949, p. 346-349). Sa consistance est objective de même que ses implications : il amène à interroger les sens

8 Ce petit texte, réponse à une enquête dirigée par Rodolphe Broda, se compose pour la plupart d'extraits de 1909a, à paraître.

9 Et nord-américaines évoquées «à titre complémentaire» (1912, p. 138). 
dans lesquels Durkheim emploie les termes de la division passé-présent, qui est l'axe de son raisonnement. Le second argument est une découverte tardive des spécialistes. On n'en parlait pas avant la lecture fonctionnaliste de Durkheim et il est vite devenu l'objet d'une surestimation. Néanmoins son appréciation est loin d'être vaine. Commençons par le premier argument, le passé délaissable.

Ce passé est celui des philosophies de l'histoire. Elles le comprennent comme une force, une vis a tergo, écrit Durkheim en adaptant un passage de sa thèse latine, qui pousse les sociétés vers une direction certaine. C'est à cette occasion que Durkheim blâme la méthode historique de Comte et l'idée de ce dernier selon laquelle la civilisation est une succession prédéterminée d'états ou stades. Si l'on s'en tient à cette conception, le passé est lié au présent par un rapport purement chronologique. Des faits se succèdent sans qu'on en voie l'origine, tandis qu'on ne rend compte d'un fait qu'à condition de le rattacher à un autre fait (1947 [1895], p. I16-II7; 1906a, p. 198). Pas d'impasses alors dans la démarche de Durkheim, car, bien qu'il lui arrive, on l'a vu, d'évoquer le passé (et le présent) en général, Durkheim ne s’intéresse qu’au passé (et au présent) de phénomènes sociaux donnés, un à la fois. Voici comment il se résume lors d'un débat de juin I9I4 à la Société française de philosophie: «Je crois et j’ai dit bien souvent que les nouveautés qui se sont produites au cours de l'évolution sociale ne sont pas un legs du passé. Le passé ne crée pas ; il ne peut que transmettre ce qui a été créé. Ses créations ne peuvent être l'œuvre que des vivants associés et coopérant, partant des contemporains. » (I9I4a, p. 69).

Comment les contemporains - associés et coopérant - suscitent-ils les changements sociaux? Par quelles causes, enfin, la sociologie explique-t-elle? S'il est question de la singularité de ces mêmes causes, cas par cas, il va de soi qu'on ne les connaîtra jamais à l'avance. Par contre, s'il s'agit de leur nature, on sait - Durkheim sait - où les chercher. Les causes des innovations qui s'opèrent au cours de l'histoire résident sans exception dans la structure morphologique ou «milieu interne» des sociétés, c'est-à-dire dans la façon dont les groupes et, au sein des groupes, les individus se disposent réciproquement, les groupes pouvant s'entremêler jusqu'à fusionner et les individus, plus ou moins nombreux, se rapprocher jusqu’à la contiguïté. Durkheim assimilant 
les groupes à des segments, selon une métaphore usitée, la fusion des groupes est aussi désignée par lui comme «coalescence» des segments sociaux. Cela d’après la «loi de gravitation du monde social», formulée dans De la Division du travail social, qui assure que les sociétés changent d’autant plus vite qu'elles sont volumineuses et densifiées (1902a [1893], p. 330, n.I). Faute de cet ancrage au milieu interne, la sociologie n'est pas en mesure de constituer des rapports de causalité : «[...] cet ordre de causes écarté, il n’y a pas de conditions concomitantes dont puissent dépendre les phénomènes sociaux» (1947 [1895], p. I15 ; voir I897b, p. 250; I899, p. I36). Le cheminement d'un phénomène social se dessine alors de la façon suivante: après qu'un mouvement du milieu interne l'a engendré, une nouvelle modification de ce milieu en change un aspect ou y ajoute quelque chose jusqu'à ce qu'une autre modification intervienne pour l'affermir ou le transformer, etc. C'est ainsi que Durkheim ne prescrit pas au hasard de comparer en tenant compte des types (ou espèces) de sociétés (comme je l’ai rappelé plus haut). Les types sociaux sont en effet identifiés par le nombre et le degré de coalescence de leurs segments (I947 [I895], p. 80-8I, 85-86).

À l'idée du passé comme contrainte et à celle, corrélative, du présent comme transition vers l'avenir («gros de l'avenir»), Durkheim oppose l'idée du passé et du présent comme fractions du temps linéaire pendant lesquelles ont lieu des événements. Cela n’est pas explicite mais la logique n’admet pas de solution de remplacement. À l'instar de la plupart des utilisateurs de la dichotomie passé-présent, Durkheim ne définit pas les termes par lesquels il indique les deux notions que cette polarité confronte (voir, par exemple, I898b, p. 37-38; I9II, p. 436; 1969b [1938], p. 21). Le principe de cohérence peut suppléer ces lacunes, suppléance d'autant plus facile que la pensée de Durkheim est systématique. Il n'y a pas une seule ligne dans l'œuvre de Durkheim qui aille contre l'évidence que tout présent est déjà passé. Le présent s'écoule au moment même où on l'observe. En revanche, tout, dans les pages de Durkheim sur la méthode de la sociologie, mène à la conclusion que le présent ne peut être un objet autonome d'étude: si l'explication des faits qui s'y produisent demande que l'on remonte à leur passé, même le plus éloigné (à leurs causes premières), une connaissance du présent séparée de celle du passé est impossible. 
Pour ce qui est du second argument de Durkheim justiciable d'un jugement d'incompatibilité avec les présupposés de sa méthode comparative, à savoir que la sociologie doit s'intéresser aux fonctions des phénomènes sociaux, le contraste résiderait dans le fait que l'explication par les causes et l'explication par les fonctions sont deux contraires. Les textes de Durkheim sont toutefois exempts d’ambiguités. Sa thèse de doctorat démarre par la question de savoir quelle est «la fonction» de la division du travail et, encore en 1909, il écrit que «les principaux problèmes de la sociologie consistent à rechercher de quelle manière s'est constituée une institution [...], quelles causes l'ont suscitée, à quelles fins utiles elle répond. » (1909a, 153). Mais la détermination des causes est prioritaire et suffit. La genèse et les progrès de la division du travail relèvent en effet de causes bien précises dont Durkheim dresse la liste (1902a [1893], Livre II). «Faire voir à quoi un fait est utile n'est pas expliquer comment il est né ni comment il est ce qu’il est. Car les emplois auxquels il sert supposent les propriétés spécifiques qui le caractérisent, mais ne le créent pas.» (1947 [1895], p. 90 ; voir aussi I895b, p. 85]). Ce que la fonction d'un phénomène peut contribuer à élucider, une fois qu’on l’a séparée de «la cause efficiente qui le produit» (1947 [1895], p. 97), c'est la vitalité ou la durée de ce même phénomène : «En effet, si l’utilité du fait n’est pas ce qui le fait être, il faut généralement qu’il soit utile pour pouvoir se maintenir.» (ibid., p. 96). C'est ainsi que dans les Formes élémentaires la fonction des rites est de renforcer la cause des croyances religieuses dont ils sont la manifestation, cette cause étant le resserrement moral des groupes. Rien mieux que le désaccord de Durkheim avec l'explication par les fonctions (le mot fonctionnalisme ne s'est pas encore propagé, mais la chose est là' ${ }^{\circ}$ ) prouve combien, d’après lui, un phénomène social est d’abord expliqué par son histoire.

10 «La plupart des sociologues croient avoir rendu compte des phénomènes une fois qu'ils ont fait voir à quoi ils servent, quel rôle ils jouent. On raisonne comme s'ils n'existaient qu'en vue de ce rôle et n'avaient d'autre cause déterminante que le sentiment, clair ou confus, des services qu'ils sont appelés à rendre. » (1947 [1895], p. 89). La formulation est presque identique in 1895b, p. 85. 


\section{4. «L'HISTOIRE SCIENTIFIQUE OU SOCIOLOGIE»}

Durkheim plaide pour ce qui semble une alliance paritaire (mais ne l'est pas) entre la sociologie et l'histoire dès ses premiers écrits (I888a, p. I08). La coopération qu'il envisage est apte à déboucher sur une unification, après une fédération temporaire.

Aucune de ces deux sciences n'aurait [...] à exercer de suprématie sur l'autre, mais il y aurait entre elles une suite d'actions et de réactions jusqu'à ce que l'équilibre, c'est-à-dire l'entente, finît par s'établir. (I888b, p. 31).

Ainsi, bien loin qu'elles soient en antagonisme, ces deux disciplines tendent naturellement l'une vers l'autre, et tout fait prévoir qu'elles sont appelées à se confondre en une discipline commune où les éléments de l'une et de l'autre se retrouveront combinés et unifiés. (I898a, p. 33).

[E]ntre l'histoire et la sociologie il n'y a pas de cloison étanche (1904, p. 242).

[H]istoire et sociologie sont deux disciplines étroitement parentes et destinées à se confondre de plus en plus. (1907, p. 244).

Nous n’avons pas à nous expliquer ici sur ce que seront dans l'avenir les rapports de la sociologie et de l'histoire; nous sommes convaincu qu'ils sont destinés à devenir toujours plus intimes et qu'un jour viendra où l'esprit historique et l'esprit sociologique ne différeront plus que par des nuances. (1909a, p. I57, n. I).

Tout ce qui rentre sous le nom d'histoire ne se prête pourtant pas à l'alliance avec la sociologie. Durkheim ne prend qu'une partie des connaissances fournies par les historiens et laisse le reste. Pourvus d'une formation littéraire (et non pas scientifique), enclins à décrire (et non pas à expliquer), les historiens, producteurs de monographies et biographies, sattachent à rapporter des faits particuliers et singuliers (guerres, luttes politiques, exploits de grands et petits personnages) mais ignorent ceux qui sont généraux et similaires, c'est-à-dire ce qu'il y a de stable et d'uniforme sous la multiplicité des situations humaines. Ils s'arrêtent à la surface, aux «incidents extérieurs» qui revêtent, cachent la constitution des sociétés; et comme ces faits, de par leur hétérogénéité et intermittence, ne sont pas susceptibles d'entrer dans des comparaisons, la sociologie n’a aucun intérêt à les retenir 
(I885, p. 348; г887, p. 340-342 ; 1947 [I895], p. 77-78; i898a, passim; I90ob, p. 33 ; 1909a, p. 155-156; Durkheim et Fauconnet, 1903, p. I46-I47). Le 28 mai I908, intervenant suite à une communication de Charles Seignobos à la Société française de philosophie, Durkheim taxe de «poussière d'événements désordonnés » le domaine habituel de l'histoire. Celui de la sociologie, composé des «manières de penser ou d’agir fixées et organisées», s'en écarte nettement (I908a, p. 212).

Mais les choses bougent même chez les adeptes de Clio. Les comparatistes y ont fait leur apparition. C'est d'abord le cas d'Albert Hermann Post, fondateur de l'histoire comparée du droit dont Durkheim a rencontré l'œuvre en Allemagne, en i886. Ce dernier y ajoute Henry Sumner Maine, Josef Kohler, Sebald R. Steinmetz et, un peu à part, Numa-Denis Fustel de Coulanges. Appartiennent au même groupe Friedrich Ratzel, qui a élevé la géographie politique au niveau d'une science comparative et explicative, des anthropologues (de Johann J. Bachofen à John F. McLennan) et des spécialistes des religions (de Max Müller - et sa mythologie comparée - à James G. Frazer). Ce qui réunit ces savants, c'est qu'ils ont tous repéré des similitudes inattendues dans l'organisation sociale de peuples éloignés les uns des autres (1887, p. 339-340; 1900b, p. 32-33; 1902b, p. 355; 1905, p. 199; Durkheim et Fauconnet, 1903, p. I46-I52). En effet, une transformation est en cours dans les sciences humaines et l'histoire en est partie prenante : le XIX ${ }^{\mathrm{e}}$ siècle a découvert les institutions, ces phénomènes bien généraux qui demeurent quand tout passe, qui «tout en évaluant, conservent leurs traits essentiels pendant de longues périodes» (Durkheim et Fauconnet, 1903, p. I47). Or, des phénomènes généraux ne peuvent qu’attester de l'existence de causes générales. Ces dernières, «partout où elles sont présentes, produisent leurs effets, toujours les mêmes, avec une nécessité égale à celle des autres causes naturelles.» (ibid., p. I52). Il en découle que, par le seul fait de s'intéresser aux institutions, «l'histoire cesse d'être une étude narrative, pour s'ouvrir à l’analyse scientifique» (ibid., p. I46). La précision apportée par Durkheim selon laquelle général et particulier signifient (aussi), respectivement, social et individuel date de sa polémique avec Gabriel Tarde. Un phénomène est général parce qu'il est social, c'est-à-dire parce qu'il s'impose aux individus, 
le contraire n'étant pas vrai, c'est-à-dire qu'il est social parce qu'il se généralise par des répétitions d’actions individuelles (1947 [1895], p. I2, n. I).

Durkheim s'adresse aux historiens voulant imiter les pionniers de la recherche comparative - prêts à souscrire à son énoncé de I887 selon lequel «il n’y a pas de science du particulier» (I888a, p. 8I I) - et leur propose l'échange de services que voici: dans un premier temps, ils se chargeront de la collecte des faits et les transmettront aux sociologues qui les passeront au crible et sépareront les faits inutiles des faits importants; les sociologues, quant à eux, enseigneront aux historiens par quels critères opérer directement la sélection des faits, ce qui fera gagner du temps. Parmi ces critères, on retiendra l'idée que les phénomènes sociaux sont assujettis à des lois (même si on ne les connaît pas encore toutes) et que leur variabilité (si l'on entreprend de les classer) est limitée. À la fin, les historiens seront devenus des sociologues et les sociologues, le cas échéant, ne dédaigneront pas de s'essayer à des travaux érudits (I888a, p. I07-I08; I888b, p. 30 ; 1947

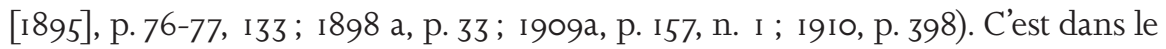
contexte de cette invitation au voyage que Durkheim formule quelques-unes de ses sentences les plus percutantes: «[...] dès qu'elle compare, l'histoire devient indistincte de la sociologie.» (I898a, 32) ; «Fustel de Coulanges aimait à répéter que la véritable sociologie, c'est l’histoire; rien n'est plus incontestable, pourvu que l'histoire soit faite sociologiquement.» (ibid., p. $33^{12}$ ); «L'histoire scientifique ou sociologie ne peut se passer de l'observation directe des faits concrets. » (1903, p. 197); «La sociologie est donc, en grand partie, une sorte d'histoire entendue d'une certaine manière.» (1909a, p. I55). Et ainsi de suite.

L'entente entre la sociologie et l'histoire que Durkheim souhaite est cependant bien loin de préfigurer un accord paritaire, tout en ne se traduisant pas par une annexion ou par une annexion seulement. Dans le plan de L’Année sociologique, la sociologie sannexe la démographie, l'économie politique, la géographie, les

II Ce rappel d'une maxime fameuse du Livre VI (chap. 2) de la Métaphysique d'Aristote n'a pas eu, en sociologie, la même fortune que la variante qu'il est usuel d'attribuer à François Simiand: «Il n'y a de science que du général».

12 Voir les dernières lignes de l'introduction de Fustel de Coulanges à L'Alleu et le domaine rural pendant l'époque mérovingienne (Paris, Hachette, 1889, p. IV-v). 
sciences de l'art, du droit, du langage, de la morale et de la religion qui deviennent toutes des sociologies spéciales. L’histoire subit-elle le même sort? Malgré le fait que Durkheim la qualifie le plus souvent de science ou discipline - et en dépit de l'hommage qu'il lui rend dans la préface du premier volume de L’Année en affirmant que la revue vise à rapprocher de la sociologie les trop nombreuses sciences qui s'en tiennent encore à l'écart, dont, en premier lieu, l'histoire («C'est surtout à l'histoire que nous pensons en parlant ainsi», I898a, p. 32) -, l'histoire n'est qu'en partie une science sociale comme les autres. Assurément la sociologie fait valoir ses créances sur cette partie. Qu’y a-t-il de plus impérialiste et de plus dévastateur que l'exhortation adressée aux historiens pour qu'ils se détournent de l'étude du particulier et embrassent uniquement celle du général? «[T] out ce qui est biographie soit des individus, soit des collectivités ${ }^{13}$ est, actuellement, sans utilité pour le sociologue.» (ibid., p. 35) ; «l'histoire ne peut être une science qu’à condition de s'élever au-dessus de l'individuel; il est vrai qualors elle cesse d'être elle-même pour devenir une branche de la sociologie.» (I903, p. 196). Que reste-t-il de l'histoire après de telles directives? Autant demander aux historiens de changer de profession ${ }^{14}$.

Mais l'histoire est aussi, voire principalement pour Durkheim, une «technique», une «science auxiliaire» - des appellations également réitérées - et c'est sous cet aspect qu'elle interfère avec la sociologie. Trois disciplines auxiliaires sont considérées par Durkheim: l’histoire, l'ethnographie et la statistique, «sans lesquelles la sociologie ne peut rien» (I897a, p. VII; voir aussi I928, p. 348). L’histoire prime l'ethnographie ${ }^{15}$. La sociologie les sollicite quand elle veut savoir quelle est l'origine des normes et des croyances, c'est-à-dire qu'elle les sollicite tout le temps puisque la plupart des manifestations de la vie sociale rentrent dans l'ordre du droit, de la morale et de la religion. Par contre, s'il est question d’apprécier le respect de ces normes, l'autorité dont jouissent ces mêmes croyances, alors la sociologie se tourne vers la statistique, du moins dans les temps modernes et relativement aux pays qui recensent des données. (190I, p. 294-295; 1909a, p. I57; 1969c [1950], p. 42-43).

13 Allusion à l'histoire des nations et des peuples.

14 C'est, grosso modo, l'une des remarques que Henri Berr (1953 [1911], p. 135, 165-168) adresse à Durkheim, qui en prend acte (1913).

I5 «[L]'histoire apporte à l'ethnographie plus de lumière qu'elle n'en reçoit» (|895a, p. 74). 
L'histoire entendue comme technique est ainsi l'érudition mise au service de la sociologie. C'est le recours à l'histoire «technique» qui fait de la sociologie la science historique que Durkheim défend encore contre Seignobos : «il n’y a pas à ma connaissance de sociologie qui mérite ce nom qui n’ait pas un caractère historique. » (1908a, p. 199 ${ }^{16}$. Dans un passage-clé de la contribution de Durkheim à De la méthode dans les sciences - passage qui résume comment se combinent chez lui les deux façons dont on peut mettre la sociologie en rapport avec l'histoire - l'histoire est un «instrument d'analyse », analogue à un outil optique largement employé dans les sciences naturelles.

[R]ien n'indique en [une institution] où commencent et où finissent les éléments divers dont elle est formée [...]. Il faut un instrument d’analyse pour les faire apparaître. C'est l'histoire qui joue ce rôle. [L]'histoire joue, dans l'ordre des réalités sociales, un rôle analogue à celui du microscope dans l'ordre des réalités physiques. /De plus, elle seule permet d'expliquer. En effet, expliquer une institution, c'est rendre compte [de ses] éléments [...], c'est montrer leurs causes [...]. Mais comment découvrir ces causes, sinon en se reportant au moment où elles ont été opérantes, c'est-à-dire où elles ont suscité les faits que l'on cherche à comprendre? Car c'est à ce moment seulement qu'il est possible de saisir la manière dont elles ont agi et engendré leur effet. Or, ce moment est derrière nous. Le seul moyen d'arriver à savoir comment chacun de ces éléments est né, c'est de l'observer à l'instant même où il est né et d’assister à sa genèse : or cette genèse a eu lieu dans le passé et, par conséquent, ne peut être connue que par l'histoire. (1909a, p. 154-155 $5^{17}$ ).

Comment séparer la sociologie de l’histoire? La réponse de Durkheim, ou, plutôt, l'apport de Durkheim à la réponse à cette question, est qu'il n’y a aucune raison de dissocier les deux disciplines et que si l'on veut vraiment s'y essayer, la voie du rapprochement de leurs objets est la plus déconseillée. À la

16 On peut considérer l'analyse de l'ouvrage de Seignobos, La Méthode historique appliquée aux sciences sociales (1902b), et l'intervention du 28 mai 1908 suite à l'exposé du même Seignobos sur «L'inconnu et l'inconscient en histoire» comme les moments notables de la (petite) participation de Durkheim au débat entre les historiens et les sociologues qui a lieu en France au tournant du XIx siècle (Prochasson, 1997; Leroux, 1998; Mucchielli, 1998, chap.12; Fournier, 2007, p.545-547, p.665-667), débat ouvert en 1894 par Paul Lacombe, avec son De l'Histoire considérée comme science et la distinction qui y est tracée entre l'étude des événements et l'étude des institutions. Ce livre ainsi que la discussion subséquente n'ont pas d'influence sensible sur les vues de Durkheim en matière d'histoire, lesquelles sont déjà bien arrêtées dès les années 1880 (Borlandi, 2012, p.26I-262).

17 Voir ce même passage, à de petites variations près, in 1908b, p. 59. 
sociologie l'étude du présent et à l'histoire celle du passé ? Mais Durkheim a argumenté que, en admettant que le présent soit observable, sa connaissance comporte celle du passé, passe par le passé. Les faits généraux de la sociologie vs les faits particuliers de l'histoire? L’histoire a pourtant su s'occuper du général bien avant l'appel de Durkheim et a continué de le faire amplement après (dans une perspective comparatiste qui plus est ${ }^{18}$ ), sans délaisser le particulier. Reste la méthode. Il suffit de poser que la sociologie explique les phénomènes sociaux par leurs fonctions ou par les relations qu'ils entretiennent les uns avec les autres - et non par leurs causes - pour que les chemins de la sociologie et de l'histoire divergent. Durkheim s'est appliqué à réduire à sa juste mesure l'explication par les fonctions (à ses yeux secondaire par rapport à celle causale). Il n'a rien dit de l'explication par les relations, n'était-ce que parce que le structuralisme, qui l’a codifiée, était encore à venir.

\section{BIBLIOGRAPHIE}

BERNARD Cl., 1984 (1865), Introduction à l'étude de la médecine expérimentale, Paris, Flammarion.

BERR H., 1953 (I9|I), La Synthèse en histoire, Paris, Albin Michel.

BORLANDI M., 2012, «Lacombe, Durkheim et le groupe de L'Année sociologique», in A. Fine et N. Adell (dir.), Histoire et anthropologie de la parenté. Autour de Paul Lacombe (1834-1919), Paris, Éditions du CTHS, p. 257-268.

COMTE A., 1975 (I830-1842), Cours de philosophie positive, Paris, Hermann, 2 vol.

DAVY G., 1925, «Vues sociologiques sur la famille et la parenté d'après Durkheim», Revue philosophique, 100, p.79-117. Repris comme «La famille et la parentée d'après Durkheim», in Id., Sociologues d'hier et d'aujourd'hui, Paris, Alcan, 1931, p. 103-157.

-, 1949, «L'explication sociologique et le recours à l'histoire d'après Comte, Mill et Durkheim », Revue de métaphysique et de morale, 54, p. 330-36l.

I8 Depuis le discours d'ouverture prononcé par Henri Pirenne à Bruxelles, au Ve Congrès international des science historiques, le 9 avril 1923: «De la méthode comparative en histoire». 
DURKHEIM É., 1885, C. r. de L. Gumplowicz, Grundriss der Soziologie (I885), Revue philosophique, 20, [p.627-634]. Repris in DURKHEIM, 1975, vol. I, p. 344-354.

-, 1887, «La science positive de la morale en Allemagne», Revue philosophique, 24, [p. 33-58, II3-142, 275-284]. Repris in DURKHEIM, 1975, vol. I, p. 267-343.

-, |888a, «Cours de science sociale. Leçon d'ouverture», Revue internationale de l'enseignement, 15, [p.23-48]. Repris in DURKHEIM, 1970, p.77-II0.

-, | 1888b, «Introduction à la sociologie de la famille», Annales de la Faculté des lettres de Bordeaux, 10, [p. 257-28I]. Repris in DURKHEIM, 1975, vol. 3, p. 9-34.

-, 1892, Quid Secundatus Politicae Scientiae Instituendae Contulerit, Bordeaux,

Gounouilhou. Traduit comme «La contribution de Montesquieu à la constitution de la science sociale», in DURKHEIM, 1953, p.25-113.

-, | 895a, «L'origine du mariage dans l'espèce humaine d'après Westermarck»,

Revue philosophique, 40, [p. 606-623]. Repris in DURKHEIM, 1975, vol. 3, p. 70-92.

-, 1895b, «Lo stato attuale degli studi sociologici in Francia», La Riforma sociale, 3,

[p.607-622, 691-707]. Traduit comme «L'état actuel des études sociologiques en France», in DURKHEIM, 1975, vol. I, p.73-108.

-, 1897a, Le Suicide. Étude de sociologie, Paris, Alcan.

-, 1897b, C. r. de A. Labriola, Essais sur la conception matérialiste de l'histoire (1896),

Revue philosophique, 44, [p. 645-65I]. Repris in DURKHEIM, 1970, p. 245-254.

-, I898a, «Préface», L’Année sociologique, I, [p. I-VII]. Repris in DURKHEIM, 1969a, p. 31-36.

-, 1898b, «La prohibition de l'inceste et ses origines», L'Année sociologique, I, [p. I-70]. Repris in DURKHEIM, 1969a, p.37-101.

-, 1899, «Préface», L'Année sociologique, 2, [p.I-VI]. Repris in DURKHEIM, 1969a, p. $135-139$.

-, 1900a, «La sociologie en France au XIX e siècle», Revue bleue, 4 s., 13, [p. 609-613, 647-652]. Repris in DURKHEIM, 1970, p. III-136.

-, 1900b, «La sociologia e il suo dominio scientifico», Rivista italiana di sociologia, 4, [p. 127-148]. Traduit comme «La sociologie et son domaine scientifique», in DURKHEIM, 1975, vol. I, p. 13-36.

-, 1901, «Introduction», L'Année sociologique, 4, [p.433-436]. Repris comme «Note sur la sociologie criminelle et la statistique morale», in DURKHEIM, 1969a, p. 293-296. 
-, 1902a [1893], De la Division du travail social, Paris, Alcan.

-, 1902b, C. r. de Ch. Seignobos, La Méthode historique appliquée aux sciences sociales (190I), L'Année sociologique, 5, [p. 123-127]. Repris in DURKHEIM, 1969a, p. 352-356.

-, 1903, C. r. de G. Salvemini, «La storia considerata come scienza», B. Croce, «La storia considerata come scienza», G. Sorel, «Storia e scienze sociali» (1902), L'Année sociologique, 6, [p. 123-125]. Repris in Durkheim, 1975, vol. I, p. 195-197.

-, 1904, «Intervention. Soutenance de thèse de G. Glotz», Revue de philosophie, 5, [p.49l-493]. Repris comme «Remarques sur l'évolution du droit criminel en Grèce», in Durkheim, 1975, vol. I, p. 24I-243.

-, 1905, «On the Relation of Sociology to the Social Sciences and to Philosophy», Sociological Papers, I, p. 197-200.

-, 1906a, C. r. de A. D. Xenopol, «Sociologia e storia» (1904), L’Année sociologique, 9, [p. 139-140]. Repris in DURKHEIM, 1975, vol. I, p. 197-199.

-, 1906b, «L'évolution et le rôle de l'enseignement secondaire en France», Revue bleue, 5e s., 5, [p.70-77]. Repris in DURKHEIM, 1966 (1922), p. $103-120$.

-, 1906c, «Observation sur la question: "Distinction et rapports de la sociologie et de l'ethnographie"»», Bulletin du Comité des travaux historiques et scientifiques - Section des sciences économiques et sociales, Paris, Imprimerie Nationale [p. 200-20I]. Repris comme «Débat sur les rapports de l'ethnologie et la sociologie», in DURKHEIM, 1975, vol. I, p. 256-258.

-, 1907, «Intervention à un débat "Sur la réforme des institutions judiciaires: l'enseignement du droit" », Libres entretiens, 3e s., [p. 361-...- 42I]. Repris comme «La sociologie et l'enseignement du droit» in DURKHEIM, 1975, vol. I, p. 243-245.

-, 1908a, «Intervention à la discussion sur "L'inconnu et l'inconscient en histoire" à la séance du 28 mai 1908 de la Société française de philosophie», Bulletin de la Société française de philosophie, 8, [p. 229-247]. Repris comme «Débat sur l'explication en histoire et en sociologie», in DURKHEIM, 1975, vol. I, p. 199-217.

- 1908b, «Contribution à une «Enquête sur la sociologie», Les Documents du progrès, 2, [p. 131-133]. Repris comme «Remarque sur la méthode en sociologie», in DURKHEIM, 1975, vol. I, p.58-61.

-, 1909a, «Sociologie et sciences sociales», in P. F. Thomas (dir.), De la méthode dans les sciences (Ire s.), Paris, Alcan [p. 259-285]. Repris in DURKHEIM, 1970, p. I37-I59. 
-, 1909b, «Sociologie religieuse et théorie de la connaissance», Revue de métaphysique et de morale, 17, p.733-758.

-, 1910, C. r. de E. Meyer, Geschichte des Altertums (1907), L'Année sociologique, II, [p. 5-13]. Repris in DURKHEIM, 1975, vol. I, p. 391-399.

-, |911, «Préface», in O. Hamelin, Le Système de Descartes, Paris, Alcan, [p. V-XI]. Repris in DURKHEIM, 1975, vol. I, p.433-438.

-, 1912, Les Formes élémentaires de la vie religieuse. Le système totémique en Australie, Paris, Alcan.

-, 1913, C. r. de H. Berr, La Synthèse en histoire (1911), L’Année sociologique, 12, [p. 26-27]. Repris in DURKHEIM, 1969a, p. 674-675.

-, 1914a, «Intervention à la discussion sur "Une nouvelle position du problème moral" à la séance du 2 juin 1914 de la Société française de philosophie», Bulletin de la Société française de philosophie, 14, [p. 26-29, 34-36]. Repris comme «Une confrontation entre bergsonisme et sociologisme», in DURKHEIM, 1975, vol. I, p.64-70.

-, 1914b, «Le dualisme de la nature humaine et ses conditions sociales», Scientia, 15, [p. 206-221]. Repris in DURKHEIM, 1970, p.314-332.

-, 1928, Le Socialisme. Sa définition, ses débuts, la doctrine saint-simonienne, Paris, Alcan.

-, 1947 (1895), Les Règles de la méthode sociologique, Paris, PUF.

-, 1953, Montesquieu et Rousseau, précurseurs de la sociologie, Paris, Rivière.

-, 1966 (1922), Éducation et sociologie, Paris, PUF.

-, 1969a, Journal sociologique, Paris, PUF.

-, 1969b (1938), L'Évolution pédagogique en France, Paris, PUF.

-, 1969c (1950), Leçons de sociologie. Physique des moeurs et du droit, Paris, PUF.

-, 1970, La Science sociale et l'action, Paris, PUF.

-, 1975, Textes, Paris, Éditions de Minuit, 3 vol.

- et P. FAUCONNET, 1903, «Sociologie et sciences sociales», Revue philosophique, 55, [p.465-497]. Repris in DURKHEIM, 1975, vol. I, p. I2I- 159.

FOURNIER M., 2007, Émile Durkheim (I858-1917), Paris, Fayard.

LAMANNA M. A., 2002, Émile Durkheim and the Family, London, Sage. 
LEROUX R., 1998, Histoire et sociologie en France. De l'histoire-science à la sociologie durkheimienne, Paris, PUF.

MILL J. S., 1974 (I843), A System of Logic Ratiocinative and Inductive, Part II, in Id., The Collected Works, Toronto, University of Toronto Press, vol. 8.

MUCCHIELLI L., 1998, La Découverte du social. Naissance de la sociologie en France (1970-1914), Paris, La Découverte.

PROCHASSON Ch., 1997, «Histoire et sociologie: Henri Berr et les durkheimiens (1900-1914)», in A. Biard, D. Bourel et E. Brian (dir.), Henri Berr et la culture du $x x^{e}$ siècle, Paris, Albin Michel, p. 62-79. 